\title{
SETTLING TIME ASSIGNMENT WITH PID
}

\author{
Nguyen Doan Phuoc, Nguyen Hoai Nam \\ Hanoi University of Science and Technology, 1 Dai Co Viet Street, Hanoi \\ "Email:nam.nguyenhoai@hust.edu.vn
}

Received: 24 October 2016; Accepted for publication: 21 February 2017

\begin{abstract}
In this paper, the settling time of closed-loop systems controlled with PID, which is established by using magnitude and symmetric optimum methods, is analyzed. These methods have some limitations such as limited frequency bands, existence of overshoot and larger settling time. Thus, our aim is to improve these performances of the closedloop system for a certain class of transfer functions. The proposed method will be tested on a DC motor by simulation. It can be concluded that the method provides a larger range of frequency, smaller settling time and no overshoot.
\end{abstract}

Keywords: PID controller, magnitude optimum criterion, symmetric optimum criterion, firstorder system, first-order plus integral system.

\section{INTRODUCTION}

Many PID tuning methods have been developed since 1940s. These methods can be classified into groups: practical approach [1 - 4]; optimal approach [5 - 8]; gain and phase margin specification based approach [9 - 13] and others [14 - 18]. However, there have not been yet any method that concurrently dealing with both the settling time and the overshoot of the closed-loop system.

It is known that when plants, having transfer functions of first-order, second-order and third-order systems the magnitude optimum method based parameter determination $k_{p}, T_{I}$ and $T_{D}$ [5 - 8] for the PID controller seems to be one of the most effective methods, validated in reality. However, there has been no fundament until now to conclude that the magnitude optimum method is the only one to provide PID controller parameters to make the closed-loop system stable and the settling time short in the sense that the closed-loop system with transfer function

$$
G_{c}(s)=\frac{R(s) G(s)}{1+R(s) G(s)}
$$

has 


$$
\Omega=\left\{\omega \in \mathrm{R}^{+}|| G_{c}(j \omega) \mid \approx 1\right\}
$$

in small range of frequency, which is large enough.

A method, proposed in this work, has an approach that the closed-loop system, obtained as follows,

$$
G_{c}(s)=\frac{1}{1+T^{\prime} s}
$$

has as small $T^{\prime}$ as possible (when $T^{\prime}$ is smaller, $\Omega$ is larger), which will demonstrate the conclusion.

\section{PID CONTROLLER DESIGN}

\subsection{Control of First-Order Systems}

Firstly, let's assume that the plant has a form of first-order system and a PI controller is used. Then, the transfer function of the plant is represented as follows

$$
G(s)=\frac{k}{1+T s}
$$

and the PI controller is

$$
R(s)=k_{p}\left(1+\frac{1}{T_{I} s}\right)=\frac{k_{p}\left(1+T_{I} s\right)}{T_{I} s}
$$

Thus, the open-loop transfer function is

$$
G_{o}(s)=R(s) G(s)=\frac{k_{p} k\left(1+T_{I} s\right)}{T_{I} s(1+T s)}
$$

and the closed-loop transfer function is

$$
G_{c}(s)=\frac{G_{o}(s)}{1+G_{o}(s)}=\frac{1+T_{I} s}{\frac{T_{I} T}{k_{p} k} s^{2}+T_{I}\left(1+\frac{1}{k_{p} k}\right) s+1}
$$

Since the first-order system (2) with unit gain is always stable, the steady state error is zero and the settling time is smaller if the time constant is shorter, in this case the two parameters $k_{p}$ and $T_{I}$ are determined such that the closed-loop transfer function (4) has the expected form as (2). In order to do that, $k_{p}$ and $T_{I}$ are chosen to cancel out a zero by a pole. The zero is $s=-1 / T_{I}$. This is equivalent to

$$
\frac{T_{I} T}{k_{p} k} s^{2}+T_{I}\left(1+\frac{1}{k_{p} k}\right) s+1=0 \text { as } s=-\frac{1}{T_{I}}
$$

or

$$
0=T-T_{I}\left(1+k_{p} k\right)+T_{I} k_{p} k
$$


Thus $T_{I}=T$.

Next, the closed-loop system becomes

$$
G_{c}(s)=\frac{1+T_{I} s}{\frac{T_{I} T}{k_{p} k} s^{2}+T_{I}\left(1+\frac{1}{k_{p} k}\right) s+1}=\frac{1}{1+T^{\prime} s}
$$

with the selected parameters as above, if the time constant $T^{\prime}$ satisfies the following equation:

$$
\begin{array}{cc} 
& \left(1+T^{\prime} s\right)\left(1+T_{I} s\right)=\left(1+T^{\prime} s\right)(1+T s)=\frac{T_{I} T}{k_{p} k} s^{2}+T_{I}\left(1+\frac{1}{k_{p} k}\right) s+1 \\
& =\frac{T^{2}}{k_{p} k} s^{2}+\frac{T\left(1+k_{p} k\right)}{k_{p} k} s+1 \\
\Leftrightarrow \quad 1+\left(T^{\prime}+T\right) s+T^{\prime} T s^{2}=\frac{T^{2}}{k_{p} k} s^{2}+\frac{T\left(1+k_{p} k\right)}{k_{p} k} s+1 & T^{\prime}=\frac{T\left(1+k_{p} k\right)}{k_{p} k}-T=\frac{T}{k_{p} k}
\end{array}
$$

This implies

$$
G_{c}(s)=\frac{1+T_{I} s}{k_{p} k\left[T_{I} T s^{2}+T_{I}\left(1+k_{p} k\right) s+1\right]}=\frac{1}{1+\frac{T}{k_{p} k} s}
$$

Hence, in the case of $T_{I}=T$ the bigger $k_{p}$ is chosen, the smaller settling time of closedloop system will be. Furthermore, the closed-loop system is stable and has no overshoot.

Obviously, in comparison to the performance of the magnitude optimum criterion method, the proposed PI controller provides a more flexible band of frequency $(\Omega)$. When the parameter $k_{p}$ is bigger, the frequency range $\Omega$ becomes wider:

$$
\lim _{k_{p} \rightarrow \infty} G_{c}(s)=1, \forall s
$$

Therefore, the desired settling time $T_{5 \%}$ of closed-loop system can be assigned by choosing

$$
k_{p}=\frac{T \ln 20}{k T_{5 \%}}
$$

which is clearly obtained from its unit step response

$$
h(t)=1-e^{-t / T^{\prime}} \text { or } 0.95=1-e^{-T_{5 \%} / T^{\prime}}
$$

\subsection{Control of Second-Order Systems}

When the plant has the form of a second-order system, we use a PID controller. The plant model 
and the controller are represented, respectively, as follows

- Plant: $G(s)=\frac{k}{\left(1+T_{1} s\right)\left(1+T_{2} s\right)}$

- PID controller: $R(s)=k_{p}\left(1+\frac{1}{T_{I} s}+T_{D} s\right)=\frac{k_{p}\left(1+T_{A} s\right)\left(1+T_{B} s\right)}{T_{I} s}$

where $T_{A}+T_{B}=T_{I}$ and $T_{A} T_{B}=T_{I} T_{D}$, it means that whenever $T_{A}$ and $T_{B}$ are known we can obtain $T_{I}$ and $T_{D}$. The open-loop system is

$$
G_{o}(s)=R(s) G(s)=\frac{k_{p} k\left(1+T_{A} s\right)\left(1+T_{B} s\right)}{T_{I} s\left(1+T_{1} s\right)\left(1+T_{2} s\right)}=\frac{k_{p}^{\prime} k\left(1+T_{A} s\right)\left(1+T_{B} s\right)}{T_{A} s\left(1+T_{1} s\right)\left(1+T_{2} s\right)}
$$

where

$$
k_{p}^{\prime}=\frac{k_{p} T_{A}}{T_{I}}
$$

Thus, if it is chosen that $T_{B}=T_{2}$ then the transfer function (5) has the same form as (3) shown in the first case

$$
G_{o}(s)=\frac{k_{p}^{\prime} k\left(1+T_{A} s\right)}{T_{A} s\left(1+T_{1} s\right)}
$$

where the parameters $T_{I}$ and $k_{p}$ are replaced by the parameters $T_{A}$ and $k_{p}^{\prime}$, respectively. So, with the set of parameters in the case 1: $T_{A}=T_{1}$ and $k_{p}^{\prime}$ arbitrary, the closed-loop transfer function becomes a first-order system with unity gain and the time constant

$$
T^{\prime}=\frac{T_{1}}{k_{p}^{\prime} k}=\frac{T_{1} T_{I}}{k k_{p} T_{A}}=\frac{T_{I}}{k k_{p}}
$$

and therefore, the bigger $k_{p}$ is chosen, the smaller its time constant $T^{\prime}$ will be. Thus, we have

$$
T_{I}=T_{A}+T_{B}=T_{1}+T_{2} \text { and } T_{D}=\frac{T_{A} T_{B}}{T_{I}}=\frac{T_{1} T_{2}}{T_{1}+T_{2}}
$$

In summary, the larger $k_{p}$ is optionally selected the smaller the settling time associated with $T^{\prime}$ will be. The assignment of desired settling time $T_{5 \%}$ for the closed-loop system can be easily realized with

$$
k_{p}=\frac{\left(T_{1}+T_{2}\right) \ln 20}{k T_{5 \%}}
$$

In addition, there is no overshoot in the closed-loop system.

\subsection{Extended Method to Integral First-Order Systems}


For the basic control theory of linear systems, it is well known that the symmetric optimum method is applied to find the parameters $k_{p}, T_{I}$ and $T_{D}$ of the PID controller

$$
R(s)=k_{p}\left(1+\frac{1}{T_{I} s}+T_{D} s\right)
$$

for integral first-order systems and integral second-order systems, in order to make the closedloop system stable and keep the phase margin larger. However, the disadvantage of the method is big overshoot. Although the overshoot can be decreased with the usage of an input filter, this causes an additional cost.

The proposed method for determination of PID parameters later will guarantee that the closed-loop system has no overshoot and arbitrary small settling time. It is similar to first-order or second-order systems, the idea of this approach is also to find parameters $k_{p}, T_{I}$ and $T_{D}$ of the PID controller for the integral first-order plant

$$
G(s)=\frac{k}{s(1+T s)}
$$

such that the closed-loop system has the following form:

$$
G_{c}(s)=\frac{1+T_{t} s}{\left(1+T_{m 1} s\right)\left(1+T_{m 2} s\right)}
$$

Consider a closed-loop system, which includes an integral first-order system (7) and a PID controller (6)

$$
R(s)=k_{p}\left(1+\frac{1}{T_{I} s}+T_{D} s\right)=\frac{k_{p}\left(1+T_{A} s\right)\left(1+T_{B} s\right)}{T_{I} s}
$$

with $T_{A}+T_{B}=T_{I}$ and $T_{A} T_{B}=T_{I} T_{D}$. The open-loop system is as follows

$$
G_{o}(s)=R(s) G(s)=\frac{k k_{p}\left(1+T_{A} s\right)\left(1+T_{B} s\right)}{T_{I} s^{2}(1+T s)}
$$

Thus, if $T_{B}=T$ then $T_{I}=T_{A}+T$ and the closed-loop system is

$$
G_{c}(s)=\frac{G_{h}(s)}{1+G_{h}(s)}=\frac{k k_{p}\left(1+T_{A} s\right)}{\left(T_{A}+T\right) s^{2}+k k_{p}\left(1+T_{B} s\right)}
$$

So, the closed-loop system will be the same as the expected form (8) if there the following equation must be satisfied

$$
\begin{gathered}
\frac{k k_{p}\left(1+T_{A} s\right)}{\left(T_{A}+T\right) s^{2}+k k_{p}\left(1+T_{A} s\right)}=\frac{1+T_{t} s}{\left(1+T_{m 1} s\right)\left(1+T_{m 2} s\right)}, \forall s \\
\Leftrightarrow k k_{p}\left(1+T_{A} s\right)\left(1+T_{m 1} s\right)\left(1+T_{m 2} s\right)=\left[\left(T_{A}+T\right) s^{2}+k k_{p}\left(1+T_{A} s\right)\right]\left(1+T_{t} s\right), \forall s
\end{gathered}
$$




$$
\begin{aligned}
& \Leftrightarrow \quad\left\{\begin{array}{l}
T_{A}\left(T_{m 1}+T_{m 2}\right)+T_{m 1} T_{m 2}=T_{A} T_{t}+\frac{T_{A}+T}{k k_{p}} \\
T_{A}+T_{t}=T_{A}+T_{m 1}+T_{m 2} \\
T_{m 1} T_{m 2} T_{A}=\frac{\left(T_{A}+T\right) T_{t}}{k k_{p}}
\end{array}\right. \\
& \Leftrightarrow\left\{\begin{array} { l } 
{ T _ { m 1 } T _ { m 2 } = \frac { T _ { A } + T } { k k _ { p } } } \\
{ T _ { t } = T _ { m 1 } + T _ { m 2 } } \\
{ T _ { m 1 } T _ { m 2 } T _ { A } = \frac { ( T _ { A } + T ) T _ { t } } { k k _ { p } } }
\end{array} \Leftrightarrow \left\{\begin{array}{l}
T_{m 1} T_{m 2}=\frac{T_{A}+T}{k k_{p}} \\
T_{A}=T_{t}=T_{m 1}+T_{m 2}
\end{array}\right.\right.
\end{aligned}
$$

This implies that

$$
\left\{\begin{array}{l}
k_{p}=\frac{T_{A}+T}{k T_{m 1} T_{m 2}}=\frac{T+T_{m 1}+T_{m 2}}{k T_{m 1} T_{m 2}} \\
T_{I}=T_{A}+T=T_{m 1}+T_{m 2}+T \\
T_{D}=\frac{T_{A} T_{B}}{T_{I}}=\frac{\left(T_{m 1}+T_{m 2}\right) T}{T_{m 1}+T_{m 2}+T}
\end{array}\right.
$$

It can be seen that with the above equations, $T_{m 1}$ and $T_{m 2}$ are freely chosen. The expected transfer function (8) becomes

$$
G_{c}(s)=\frac{1+\left(T_{m 1}+T_{m 2}\right) s}{\left(1+T_{m 1} s\right)\left(1+T_{m 2} s\right)}
$$

Thus, with an addition of an input filter as illustrated in Fig.1:

$$
M(s)=\frac{1}{1+\left(T_{m 1}+T_{m 2}\right) s}
$$

to obtain the closed-loop system:

$$
G_{c}(s) M(s)=\frac{1}{\left(1+T_{m 1} s\right)\left(1+T_{m 2} s\right)}
$$

we always can choose $T_{m 1}$ and $T_{m 2}$ such that this closed-loop system has a settling time $T_{5 \%}$ as desired. Obviously, there are two possible combinations of time constants $T_{m 1}$ and $T_{m 2}$. The first case is $T_{m 1}=T_{m 2}=T_{m}$ and the second one is $T_{m 1} \neq T_{m 2}$.

Now some steps, about how to choose these time constants such that the desired settling time can be achieved, will be shown. Firstly,

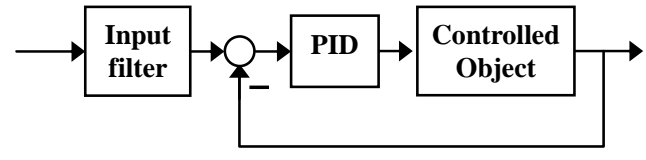

Figure 1. Control with PID and input filter. 
let's consider the case one. With the usage of input filter as shown in Fig. 1, the closed-loop transfer function is as follows

So, the unit step response is

$$
G_{c}(s)=\frac{1}{\left(1+T_{m} s\right)^{2}}
$$

$$
h(t)=1-e^{-t / T_{m}}-\frac{t}{T_{m}} e^{-t / T_{m}}
$$

Thus, the desired settling time $T_{5 \%}$ will be the solution to the following equation

$$
e^{-t / T_{m}}+\frac{t}{T_{m}} e^{-t / T_{m}}-0.05=0
$$

Let $x=T_{5 \%} / T_{m}$. Then

$$
f(x)=e^{-x}(1+x)-0.05=0
$$

As $x=0, f(0)=0.95$ and when $x=\infty$ it is obtained $f(\infty)=-0.05$. In addition, $f^{\prime}(x)=-e^{-x} x<0, \forall x>0$. That means there must be a unique solution to the equation (10). The solution is approximately equal to 4.7439 . Hence,

$$
T_{m 1}=T_{m 2}=T_{m}=\frac{T_{5 \%}}{4.7439}
$$

Then, the PID parameters are determined from Eq. (9).

For the second case, the closed-loop system with input filter is

$$
G_{c}(s)=\frac{1}{\left(1+T_{m 1} s\right)\left(1+T_{m 2} s\right)}
$$

Without loss of generality, let $T_{m 1}=k_{s} T_{m 2}$ where $0<k_{s}<1$. Doing the same procedure as the first case, the following equation is obtained

$$
\frac{T_{m 1} e^{-t / T_{m 1}}-T_{m 2} e^{-t / T_{m 2}}}{T_{m 1}-T_{m 2}}-0.05=0
$$

Obviously, the expected settling time will be the solution to the equation (12). Let $x=T_{5 \%} / T_{m 2}$, then substitute it and $T_{m 1}$ into the equation (12) to have

$$
f\left(x, k_{s}\right)=\frac{k_{s} e^{-x / k_{s}}-e^{-x}}{k_{s}-1}-0.05=0
$$

It can be easily verified that

$$
f\left(0, k_{s}\right)=0.95, f\left(\infty, k_{s}\right)=-0.05 \text { and } f^{\prime}\left(x, k_{s}\right)<0 .
$$

This implies the equation (13) must have only one solution with respect to $k_{s}$. The Tab. 1 shows solutions for some values of $k_{s}$.

Table 1. Solution with respect to $k_{s}$.

\begin{tabular}{|c|c|c|c|c|c|}
\hline$k_{s}$ & 0.01 & 0.3 & 0.5 & 0.7 & 0.99 \\
\hline$x^{*}$ & 3.005 & 3.352 & 3.6761 & 4.067 & 4.7202 \\
\hline
\end{tabular}


Thus,

$$
T_{m 2}=\frac{T_{5 \%}}{x^{*}} \text { and } T_{m 1}=k_{s} \frac{T_{5 \%}}{x^{*}} .
$$

Hence, given a desired settling time, first we have to choose $k_{s}$, then time constants $T_{m 1}$ and $T_{m 2}$ are determined by using Eq. 14, and finally the PID parameters are calculated from Eq. 9. It can be concluded that as $k_{s} \rightarrow 1$ then $x^{*} \rightarrow 4.7439$. Thus, the second case becomes the first case.

\section{SIMULATION RESULTS}

\subsection{Motor Speed Control}

Given a speed transfer function of a DC motor as follows [19]

$$
G(s)=\frac{k}{1+T s}
$$

where $k=100, T=1.9568$, the input is the current applied to the DC motor and the output is the speed of the rotor. The I controller parameter is determined by using the magnitude optimum method is $T_{I}=2 k T=391.4$, whereas the proposed method provides values for the PI controller as $T_{I}=T=1.9568$ and $k_{p}=0.02\left(k_{p}=0.1\right.$ for the second case $)$ which can be arbitrarily chosen such that the settling time is smaller than any expected value. Simulation results for two cases are shown in Fig. 2 and Fig. 3. For the first case, the reference is a step function $r=0.5 \mathrm{rad} / \mathrm{s}$, but for the second case, the reference is a sine function $r=0.5 \sin (0.3 t)$. It can be seen that the proposed method gives much better performances in terms of settling time and overshoot. Especially, when the reference signal is a sine function, the magnitude optimum method failed to keep the stability and other performances of the close-loop system.

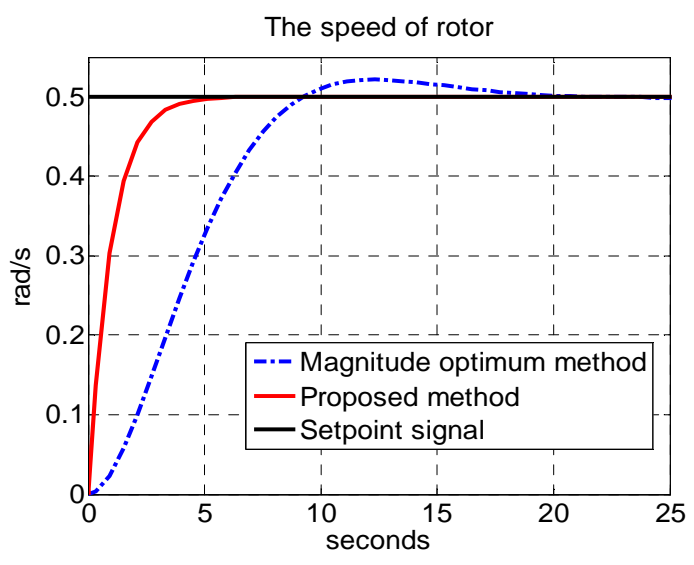

Figure 2. Responses to a step function.

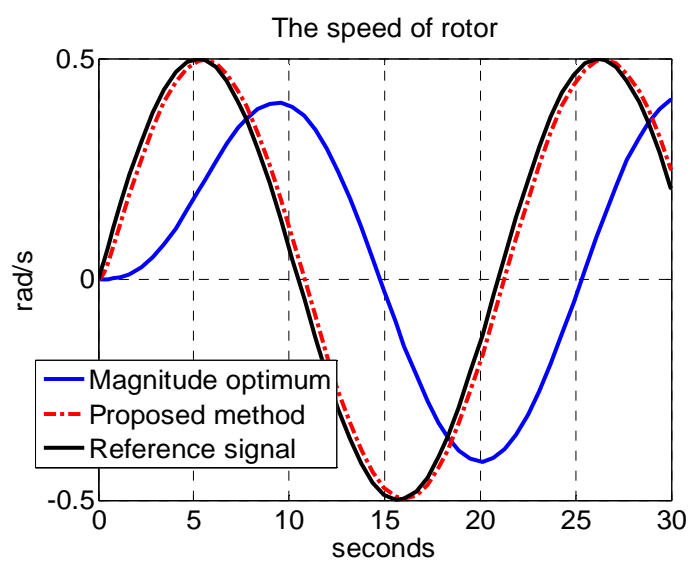

Figure 3. Responses to a sine function.

To compare performances obtained by using different methods, the $\mathrm{IAE}_{\mathrm{s}}$ (integral absolute error) at a set point change and $\mathrm{IAE}_{\mathrm{d}}$ at process disturbance change are used [4]. $\mathrm{IAE}_{\mathrm{s}}$ is calculated as follow 


$$
\mathrm{IAE}_{s}=\int_{t_{i}}^{t_{f}}|e(t)| d t
$$

where $e$ is the error between the plant output and the set point, $t_{i}$ is the initial time, which is the time of the step change, $t_{f}$ is an appropriate final time. For the $\mathrm{IAE}_{\mathrm{d}}$, the formula is the same as Eq. (15) but the initial time will be the time of a disturbance change.

The $\mathrm{IAE}_{\mathrm{s}}$ and $\mathrm{IAE}_{\mathrm{d}}$ for the two cases are shown in Tab. 2. A step disturbance with amplitude of 0.01 is applied to the input of the plant at the time of 30 seconds to measure the $\mathrm{IAE}_{\mathrm{d}}$. In conclusion, the proposed method provides better performances in term of disturbance compensation and set point change for both cases.

Table 2. IAE and $\mathrm{IAE}_{\mathrm{d}}$ for the two cases.

\begin{tabular}{|l|c|c|c|c|}
\hline \multirow{2}{*}{\multicolumn{1}{|c|}{ Methods }} & \multicolumn{2}{c|}{ Case 1} & \multicolumn{2}{c|}{ Case 2 } \\
\cline { 2 - 5 } & IAE & IAE $_{\mathrm{d}}$ & IAE & IAE $_{\mathrm{d}}$ \\
\hline \hline Magnitude Optimum & 2.23 & 4.265 & 9.254 & 15.046 \\
\hline Proposed Method & 0.4892 & 0.9788 & 0.5405 & 0.7585 \\
\hline
\end{tabular}

\subsection{Rotor Position Control}

In this section, the PID controllers are designed to control the angular position of the rotor [19] which has transfer function as below

$$
G(s)=\frac{k}{s(1+T s)}
$$

where the values of parameters are the same as in the previous section. The PI controller parameters are chosen by using the symmetric optimum method as follows

$$
a=9, T_{I}=9 a=17.61 \text { and } k_{p}=\frac{1}{k T \sqrt{a}}=0.0017
$$

but our method gives

$$
\begin{aligned}
& k_{p}=\frac{T+T_{m 1}+T_{m 2}}{k T_{m 1} T_{m 2}}=0.1575, T_{I}=T_{m 1}+T_{m 2}+T=2.8, \\
& T_{D}=\frac{\left(T_{m 1}+T_{m 2}\right) T}{T_{m 1}+T_{m 2}+T}=0.5893
\end{aligned}
$$

where the desired settling time $T_{5 \%}=2$ seconds and $T_{m 1}=T_{m 2}=0.4216$. For the second case, if $k_{s}=0.5$ and $T_{5 \%}=2$, then $T_{m 2}=0.5441$ and $T_{m 1}=0.2720$. Thus, $k_{p}=37.4724$, $T_{I}=2.7729$ and $T_{D}=0.5759$.

For zero overshoot, an input filter $M(s)$ is used to cancel the zero of the close-loop system out. The two proposed PID controllers provide similar performances as shown in Fig. 4. The results show that the proposed method gives better performances, such as smaller settling time as desired. When parameters $T_{m 1}$ and $T_{m 2}$ increase the settling time is larger but the 
overshoot is smaller and vice versa. The $\mathrm{IAE}_{\mathrm{s}}$ and $\mathrm{IAE}_{\mathrm{d}}$ are shown in Tab. 3, where a step disturbance with amplitude of 0.01 is applied to the input of the plant at the time of 70 seconds to measure the $\mathrm{IAE}_{\mathrm{d}}$. Again, the $\mathrm{IAE}_{\mathrm{s}}$ and $\mathrm{IAE}_{\mathrm{d}}$ proved that the proposed method gives much better performance than the symmetric optimum method.

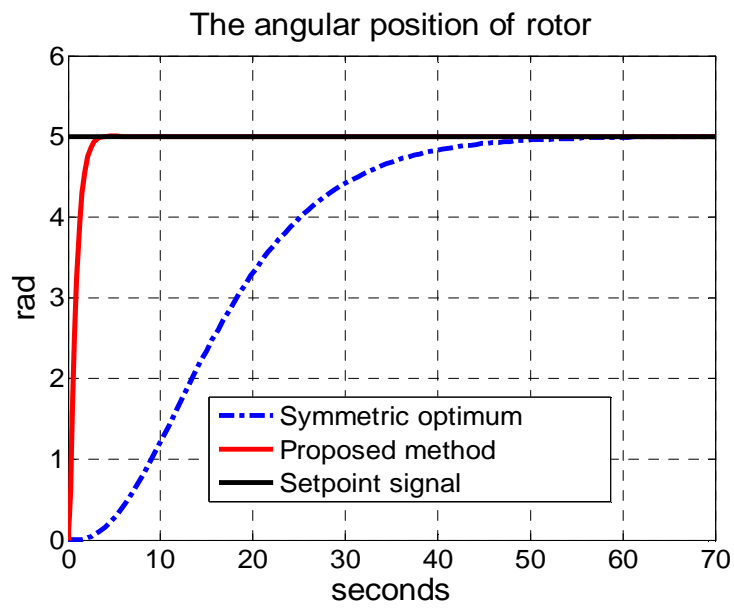

Figure 4. Responses to a step function.

Table 3. $\mathrm{IAE}_{\mathrm{s}}$ and $\mathrm{IAE}_{\mathrm{d}}$.

\begin{tabular}{|l|c|c|}
\hline \multirow{2}{*}{ Methods } & \multicolumn{2}{|c|}{ Performance } \\
\cline { 2 - 3 } & IAE $_{\mathrm{s}}$ & IAE $_{\mathrm{d}}$ \\
\hline \hline Symmetric Optimum & 24.66 & 104.94 \\
\hline Proposed Method & 0.7858 & 0.2080 \\
\hline
\end{tabular}

\section{CONCLUSIONS AND FUTURE WORKS}

In the work, a PID design method with desired settling time is proposed for plants with transfer functions of the following types: first-order system, second-order system and integral first-order system. The method provides better performances than both the magnitude optimum method and the symmetric optimum method in the sense of overshoot and settling time. In addition, the settling time can be achieved as expected for a larger frequency band. Some examples for DC motor control are used to illustrate the method.

Future works will focus on robustness, effects of model error and other types of transfer functions, may be higher-order system with time delay.

\section{REFERENCES}

1. Ziegler J. G. and Nichols N. B. - Optimum settings for automatic controllers, Trans. ASME 64 (1942) 759-765.

2. Oldenbourg R. C. and Hans S. - A uniform approach to the optimum adjustment of control loops, Trans. ASME 18 (1954) 1-9.

3. Hang C. C., Astrom K. I. and Ho W. K. - Refinements of the Ziegler-Nichols tuning formula, IEE Proc. D. 138 (1991) 111-118. 
4. Finn H. and Bernt L. - Relaxed Ziegler-Nichols Closed-Loop Tuning of PI Controllers, Modeling, Identification and Control 34 (2013) 83-97.

5. Kessler C. - Das symmetrische optimum, Regelungstechnik 11 (1958) 395-400.

6. Jeffrey W. U. and Mohammed S. - Magnitude and symmetric optimum criterion for the design of linear control systems: What is it and how does it compare with the others? IEEE Trans. Indus. Appl. 26 (1990) 489-497.

7. Konstantinos G. P. and Nikolaos I. M. - Extending the symmetrical optimum criterion to the design of PID type-p control loops, Journal of Process Control 22 (2012) 11-25.

8. Konstantinos G. P., Nikolaos D. T. and Nikolaos I. M. - Revisiting the magnitude optimum criterion for robust tuning of PID type-I control loops, Journal of Process Control 22 (2012) 1063-1078.

9. Astrom K. J. and Hagglund T. - Automatic tuning of simple regulators with specifications on phase and amplitude margins, Automatica 20 (1984) 645-651.

10. Weng K. H., Chang C. H. and Lisheng S. C. - Tuning of PID controllers based on gain and phase margin specifications, Automatica 31 (1995) 497-502.

11. Sheng Y. C. and Ching C. T. - Tuning of PID controllers based on gain and phase margin specification using fuzzy neural network, Fuzzy Sets and Systems 101 (1999) 21-30.

12. Cominos P. and Munro N. - PID controllers: recent tuning methods and design to specification, IEE Proc. Control Theory Appl. 149 (2002) 46-53.

13. Keyu L. - PID tuning for optimal closed-loop performance with specified gain and phase margins, IEEE Trans. Control Syst. Tech. 21 (2013) 1024-1030.

14. Tyreus B. D. and Luyben W. L. - Tuning PI controllers for integrator/dead time processes, Ind. Eng. Chem. 31 (1992) 2626-2628.

15. Astrom K. J. and Hagglund T. - PID controllers: Theory, design and tuning, ISA 1995.

16. Astrom, K. J. and Hagglund T. - Advanced PID control, The Instrumentalion, Systems, and Automation Society, 2005.

17. Phuoc, N. D. - Linear Control Theory, Science and Technology Publisher, 2007.

18. Ntogramatzidis L. and Ferrante A. - Exact tuning of PID controllers in control feedback design, IET Cont. Theo. Appl. 5 (2011) 565-578.

19. Siri W. and El S. - Identification and control of a DC motor using back-propagation neural networks. IEEE Transactions on Energy Conversion 6 (1991) 663-669. 